\title{
Thermal Characterization of In-Situ Materials Using Modal Reduced Models
}

\author{
Ana Gabriela Chavez Castillo ${ }^{1}$, Yassine Rouizi ${ }^{1}$, Olivier Quemener $^{1}$, Laurent Marmoret ${ }^{2}$, Yann Billaud $^{3}$ \\ ${ }^{1}$ Laboratoire de Mécanique et d'Energétique d'Evry, Université d'Evry Val d'Essonne \\ Chemin de la Tuilerie, 91220 Brétigny sur Orge, France \\ a.chavez@iut.univ-evry.fr; y.rouizi@iut.univ-evry.fr; o.quemener@iut.univ-evry.fr \\ ${ }^{2}$ Laboratoire des Technologies Innovantes, Université de Picardie \\ 80025 Amiens, France \\ ${ }^{3}$ Institut Prime CNRS, Université de Poitiers, ISAE-ENSMA, Téléport 2 \\ 11 Boulevard Marie et Pierre Curie, BP 30179, 86962 Futuroscope Chasseneuil Cedex
}

\begin{abstract}
The analytical models used by commercial probes are no longer suitable for insulating materials. Indeed, these analytical laws do not allow the taking into account of the inertia of the probe and thermal flows that exist along the sensor. The solution is then to use an inverse problem technique in which a numerical model is used, in order to be able to take into account the totality of the different thermal phenomena, in an iterative process. However, in the case of configurations that require large numerical models, this type of technique can lead to very large computation times. The modal-type reduced models can efficiently decrease the computation time, without compromising the precision of the results. In order to verify the feasibility of this argument, we conducted a study where we use a modal reduced model in a measurement procedure to determine the thermal characteristics of a material by simply inserting a probe into a wall. This purely numerical study presents the influence of the order of the reduced model, the sensitivity of the probe, and the noise on the precision of the measurement.
\end{abstract}

Keywords: Characterization, Construction Materials, Reduced Models, Identification, Measurements.

\section{Introduction}

In the field of construction, identifying the in-situ evolution of the thermal properties of materials, including insulators of bio-sourced origin, remains a current issue. We propose a technique for identifying thermal conductivity and heat capacity by inserting a simple probe in the material to be identified.

This paper presents an identification method that uses modal-type reduced models, allowing an accurate description of the geometry of the probe and taking into account all the thermal phenomena.

\section{Problem Statement}

A simple solution for in-situ identification of thermal conductivity is the use of probes that use the hot wire technique [1]. The Hukseflux probe (Figure 1) is particularly appreciated for its robustness which, by the use of a simple analytical model, allows the immediate determination of the thermal conductivity. Unfortunately the latest work on this subject has shown the limits of this technique [2] since, in the case of insulators, we cannot neglect the inertia of the probe or its high conductivity.

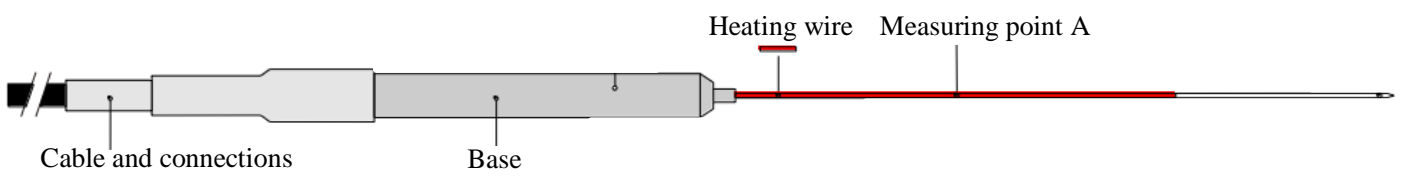

Fig. 1: TP02 Hukseflux probe. 


\subsection{Description of the Hot Wire Type Probe}

Consider the problem represented in Fig. 2 whereby the probe $\Omega_{C}$ is inserted into the insulator $\Omega_{I}$, which rests on a simple support structure $\Omega_{S}$.

The contact resistance between $\Omega_{C}$ and $\Omega_{I}$ is negligible compared to the high resistivity of the insulation. The probe consists of different materials (Figure 3), including a $\Omega_{C 3}$ resistance that dissipates a power density of $\pi=37,77 \cdot 10^{6} \mathrm{~W} \cdot \mathrm{m}^{-3}$ It consists of a temperature measurement at point $\mathrm{A}$. The set of problem parameters is specified in Table 1 . The geometry is axisymmetric, i.e. T (r, z). An environment characterized by a heat transfer coefficient of $h=10 \mathrm{~W} \cdot \mathrm{m}^{-2} \cdot \mathrm{K}^{-1}$ for a temperature of $T_{f}=16,18^{\circ} \mathrm{C}$ is considered.

\subsection{Numerical Model}

The thermal problem is expressed as a variational expression (Eq. 1). We note the domain $\Omega=\Omega_{C} \cup \Omega_{S}$, $\square \square$ is the boundary of the whole domain studied, $g$ is the test function, $c$ is the heat capacity, $k$ is the thermal conductivity, $h$ is the heat transfer coefficient, and $\pi$ is the heat source:

$$
\begin{aligned}
c_{I} \int_{\Omega_{I}} \frac{\partial T}{\partial t} g d \Omega+\int_{\Omega} c \frac{\partial T}{\partial t} g d \Omega= & -k_{I} \int_{\Omega_{I}}^{\mathrm{r}} \nabla T . \stackrel{\mathrm{r}}{\nabla} g d \Omega-\int_{\Omega} k \stackrel{\mathrm{r}}{\nabla} T . \stackrel{\mathrm{r}}{\nabla} g d \Omega-\int_{\Gamma} h T g d \Gamma \\
& +\int_{\Gamma} h T_{f} g d \Gamma+\int_{\Omega_{C 3}} \pi g d \Omega
\end{aligned}
$$

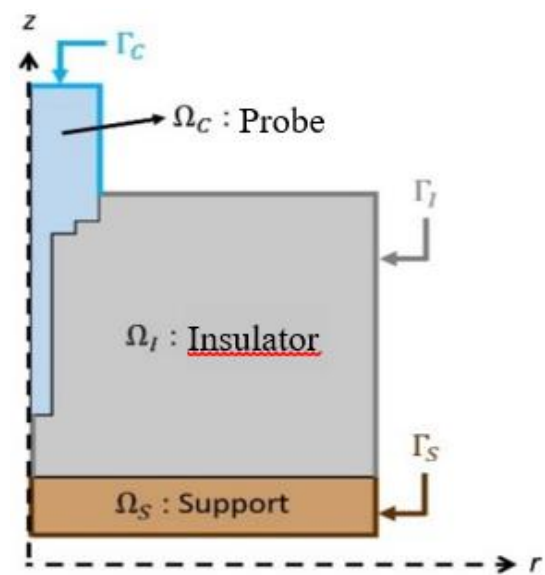

Fig. 2: Representation of the physical problem

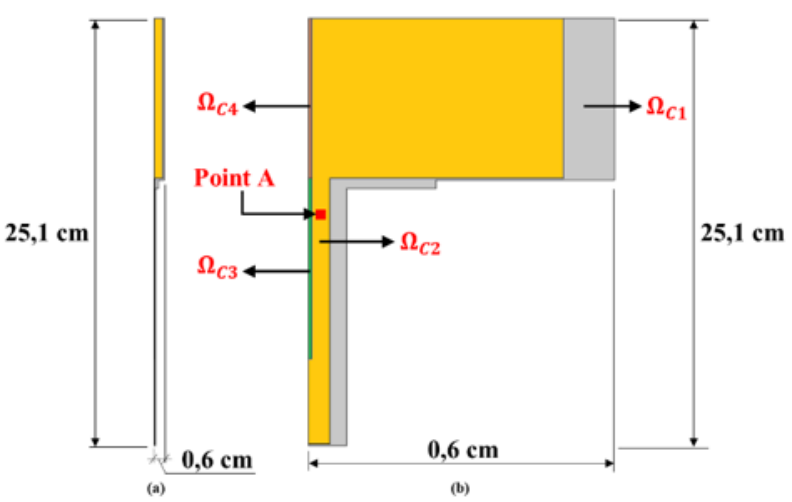

Fig. 3: Representation of probe components (a) exact proportions (b) dilated radial scale 
Table 1: Component features.

\begin{tabular}{|c|c|c|c|c|c|c|}
\cline { 4 - 7 } \multicolumn{1}{c|}{} & Insulator & Support & \multicolumn{4}{|c|}{ Probe $\Omega_{C}=\Omega_{C 1} \cup \Omega_{C 2} \cup \Omega_{C 3} \cup \Omega_{C 4}$} \\
\cline { 4 - 7 } \multicolumn{1}{c|}{} & $\Omega_{I}$ & $\Omega_{S}$ & $\Omega_{C 1}:$ Steel & $\Omega_{C 2}:$ Resin & $\Omega_{C 3}:$ Resistance & $\Omega_{C 4}:$ Cords \\
\hline$k\left[\mathrm{~W} \cdot \mathrm{m}^{-1} \cdot \mathrm{K}^{-1}\right]$ & $k_{I}$ & 0,2 & 16 & 0,30 & 19,5 & 400 \\
\hline$c\left[\mathrm{~J} \cdot \mathrm{m}^{-3} \cdot \mathrm{K}^{-1}\right]$ & $c_{I}$ & $1,97.10^{-6}$ & $3,95.10^{-6}$ & $1,28.10^{-6}$ & $3,38.10^{-6}$ & $3,42.10^{-6}$ \\
\hline
\end{tabular}

A P1 type finite element discretization leads to a matrix system (Eq. (2)) whose size is characterized by the number of nodes $N$ of the mesh, with $N \approx 20000$. Calculations are performed by a laboratory code in $\mathrm{C}++$.

$$
\left(c_{I} \mathbf{C}_{\mathbf{I}}+\mathbf{C}\right) \dot{\mathbf{T}}=\left(k_{I} \mathbf{K}_{\mathbf{I}}+\mathbf{A}\right) \mathbf{T}+\mathbf{U}
$$

\section{Modal Reduced Model}

\subsection{Presentation of the Technique}

The principle of modal reduction methods is to decompose the thermal field $T(M, t)$ over a small number $n$ of known spatial functions $\tilde{V}_{i}(M)$, which then makes it possible to obtain an estimate $\tilde{T}(M, t)$ :

$$
\tilde{T}(M, t) \approx \sum_{i=1}^{n<<N} \tilde{x}_{i}(t) \tilde{V}_{i}(M)
$$

where $\tilde{x}_{i}(t)$ are the unknown excitation states of these spatial functions. If the number of functions $n$ is small with respect to $N$, the problem is reduced. The next step is to determine the $n$ excitation states $x_{i}(t)$ compared to $N$ temperature changes $T(M, t)$. For this study, the BERM method $[3,4]$ was used, which is broken down into three stages:

- The complete computation of the N so-called Branch vectors (Eqs. (4) and (5)), which forms a basis for any type of thermal problem (Eq. (1)), including when the different parameters of the equation vary:

$$
\begin{array}{ll}
\forall M \in \Omega \cup \Omega_{I}, & k_{m} \vec{\nabla}\left(\vec{\nabla} V_{i}\right)=\mathrm{z}_{i} \mathrm{c}_{m} \mathrm{~V}_{i} \\
\forall M \in \Gamma, & k_{m} \vec{\nabla} V_{i} \cdot \overrightarrow{\mathrm{n}}=-\mathrm{z}_{i} \zeta \mathrm{V}_{i}
\end{array}
$$

where $k$ and $c$ are simple space functions and the index $m=(1, \ldots, 6)$ corresponds to each material of the domain.

The boundary condition (Eq. (5)) is an abstract condition that involves the eigenvalue $z_{i}$ of the $V_{i}$ mode. The number of Steklovs $\zeta$ ensures the dimensional homogeneity of the boundary condition and avoids the degeneration of the branch modal problem [4], that is to give a comparable importance to the two equations of the system (4) and (5):

$$
\zeta=\frac{\int c d \Omega}{\Omega_{I} \cup \Omega_{S}}
$$

- The reduction of the base is carried out by the amalgam method. It consists of reconstructing $n$ new modes $\tilde{V}_{j}$ by linear combinations of the initial modes $V_{i}$ :

$$
\forall j \in\{1, n\} \quad \tilde{V}_{j}=\sum_{p=1}^{\tilde{N}_{j}} \alpha_{j, p} V_{j, p} \quad\left(\text { with } \tilde{N}_{j} \text { such as } N=\sum_{j=1}^{n} \tilde{N}_{j}\right)
$$


The choice of this combination is then based on a reference case for which the excitation states $x_{i}(t)$ associated with the initial complete base are known. From these coefficients the distribution of the set of modes $V i$ on the reduced basis $\widetilde{V}_{j}$ is made, as well as the calculation of the weighting factors $\alpha_{j, p}$.

- The reduced problem is defined by the state equation obtained by projecting the heat equation (Eq. 1) onto the reduced basis. By noting $\tilde{\mathbf{V}}$ as the matrix comprising the entirety of the $n$ reduced modes $\widetilde{V}_{j}$ expressed in discrete form, and $\widetilde{\mathbf{X}}$ as the vector of the $n$ associated excitation states, we have:

$$
\left(c_{I} \widetilde{\mathbf{V}}^{t} \mathbf{C}_{\mathbf{I}} \widetilde{\mathbf{V}}+\widetilde{\mathbf{V}}^{t} \mathbf{C} \widetilde{\mathbf{V}}\right) \dot{\tilde{\mathbf{X}}}=\left(k_{I} \widetilde{\mathbf{V}}^{t} \mathbf{K}_{\mathbf{I}} \widetilde{\mathbf{V}}+\widetilde{\mathbf{V}}^{t} \mathbf{A} \widetilde{\mathbf{V}}\right) \widetilde{\mathbf{X}}+\widetilde{\mathbf{V}}^{t} \mathbf{U}
$$

Expressing Eq. (8) is a more compact form, we have:

$$
\left(c_{I} \mathbf{L}_{\mathbf{I}}+\mathbf{L}\right) \dot{\widetilde{\mathbf{X}}}=\left(k_{I} \mathbf{M}_{\mathbf{I}}+\mathbf{M}\right) \widetilde{\mathbf{X}}+\mathbf{N}
$$

This relationship has $n$ degrees of freedom. Therefore it is quickly solved, and then provides access to the entire temperature field by the relation (Eq. (3)).

\subsection{Reduction Results}

Since the thermal parameters of the insulator are unknown and are to be identified by this reduced model (Eq. (9)), we then choose the values of the thermal conductivity $k_{I}$ and the heat capacity $c_{I}$ of the insulator for the calculation of the Branch base and the reference model used during the reduction by Amalgam. These values are different from the values that will subsequently be identified as the exact values. The following characteristics of the insulation are chosen for the construction of the reduced model:

$$
\text { case }_{\text {base }}: k_{\text {base }}=0,01 \mathrm{~W} \cdot \mathrm{m}^{-1} \cdot \mathrm{K}^{-1} \text { et } c_{\text {base }}=25000 \mathrm{~J} \cdot \mathrm{m}^{-3} \cdot \mathrm{K}^{-1}
$$

The Steklov's number (Eq. (6)) is then: $\zeta=5580 W \cdot m^{-2} \cdot K^{-1}$. Model simulation is then performed, which is compared to the complete model for two configurations:

- the reference configuration: case $_{\text {base }}$

- a different configuration, which we choose identical to the one we will seek to identify:

$$
\text { case exact: } k_{\text {exact }}=0,0306 \mathrm{~W} \cdot \mathrm{m}^{-1} \cdot \mathrm{K}^{-1} \text { et } c_{\text {exact }}=30878 \mathrm{~J} \cdot \mathrm{m}^{-3} \cdot \mathrm{K}^{-1}
$$

Figure 4 shows the evolution of the mean errors $\bar{\varepsilon}$ and quadratic errors at the measurement point $\varepsilon_{A}$, of the reduced model compared to the complete model for these two configurations during the time $\Delta t$ of the simulation. We thus define:

$$
\begin{gathered}
\bar{\varepsilon}=\frac{1}{\Delta t . V_{\Omega \cup \Omega_{I}}} \int_{\Delta t} \int_{\Omega \cup \Omega_{I}}|\tilde{T}(M, t)-T(M, t)| d \Omega d t \\
\varepsilon_{A}=\sqrt{\frac{1}{\Delta t} \int_{\Delta t}\left(\tilde{T}_{A}(t)-T_{A}(t)\right)^{2} d t}
\end{gathered}
$$

It is noted that there is an exponential decay for the mean error, unlike the localized error at the measurement point. Indeed, the reduction process minimizes the average error. In the case where a simulation is carried out for a problem in which the insulation has the same characteristics as those used for the construction of the reduced model, the latter is very effective, since an order of $n=50$ modes leads to an error at the measuring point of the order of $\varepsilon_{A}=0,01^{\circ} \mathrm{C}$. However, for very different insulator characteristics, the errors increase by an order of magnitude: always for $n=50$ modes, we obtain $\varepsilon_{A}=0,1^{\circ} \mathrm{C}$. Here we see the importance of choosing the appropriate reference case to be used in the amalgam procedure. The identification procedure model will then depend on the accuracy of the measurement and the sensitivity of the probe with regards to the quantities identified. 

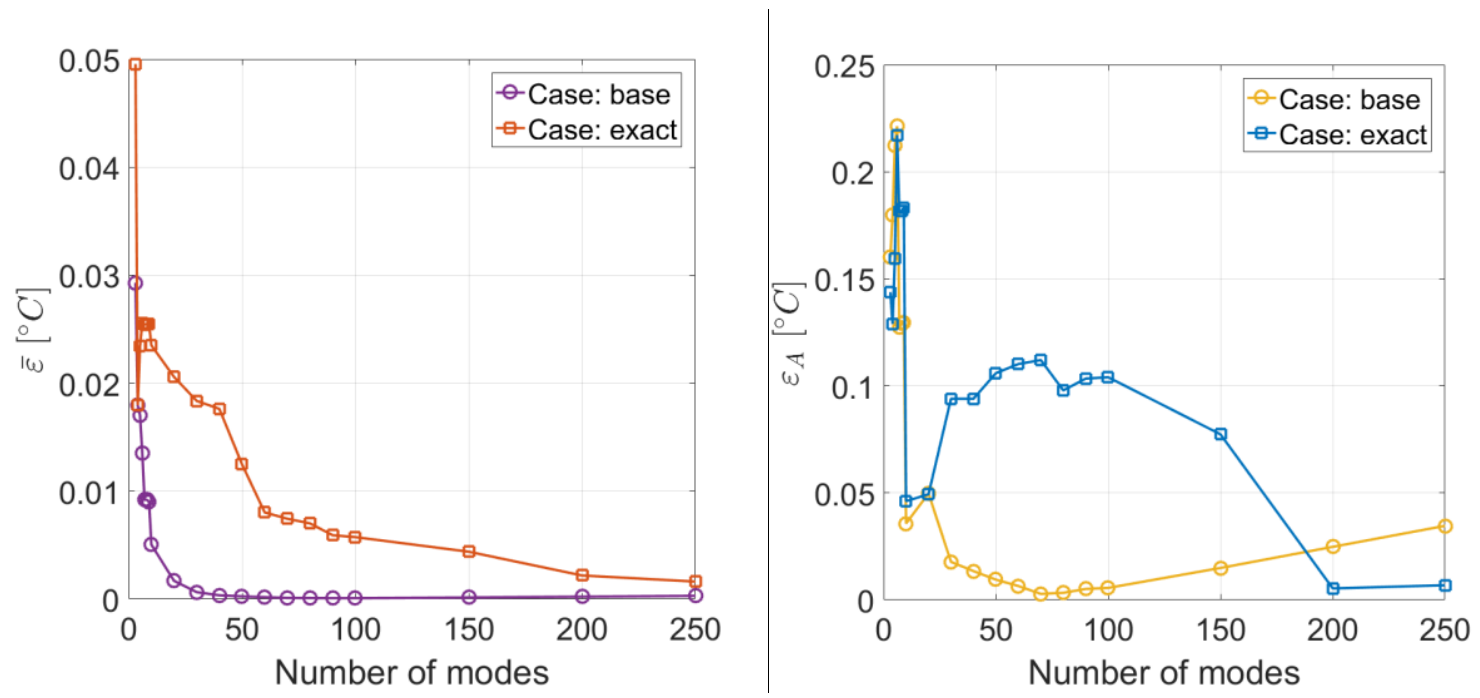

Fig. 4: Mean error $\bar{\varepsilon}$ and error at the measurement point $\varepsilon_{A}$ of the reduced model.

\section{Identification Technique}

\subsection{Principle}

The objective is to identify the parameter values of thermal conductivity $k_{I}$ and heat capacity $c_{I}$ of the insulator in which the measurement probe is inserted. The latter is provided with a single measuring point (point A in Fig. 3), which corresponds to the observable $\mathbf{Y}$, and which is connected to the temperature field $\mathbf{T}$ via an observation matrix $\mathbf{E}$. Given the size of the discrete problem, it is possible to use the modal formulation presented previously to reduce the size of the inverse problem:

$$
\mathbf{Y}=\mathbf{E} \mathbf{T}=\mathbf{E} \widetilde{\mathbf{V}} \widetilde{\mathbf{X}}
$$

The iterative identification process is based on the minimization of a quadratic criterion built on the difference between the measurement $Y\left(t_{i}\right)$ at the point A (calculated with the complete model (Eq. 2) with added noise and the result of the simulation $\hat{Y}$ performed by the reduced model (Eqs. (3), (9) and (10)) for the estimated parameters $\hat{\mathrm{k}}_{\mathrm{I}}$ and $\hat{\mathrm{c}}_{\mathrm{I}}$ at each iteration:

$$
\frac{1}{2} \sum_{i=1}^{n_{m e s}}\left(Y\left(t_{i}\right)-\hat{Y}\left(t_{i}, \hat{k}_{I}, \hat{c}_{I}\right)\right)^{2}
$$

This minimization procedure uses a non-linear programming method based on a trusted region algorithm (offered by Matlab(R), the principle of which is to replace the initial optimization problem with a series of sub-problems, which are simpler to solve.

\subsection{Sensitivity Analysis}

Because of the reduced sensitivities of the measurement to the variations of thermal conductivity and heat capacity (Eq. (14)), a sensitivity study is conducted to ensure the relevance of the measurement point used to carry out the identification.

$$
S_{c}^{*}=c \frac{\partial Y}{\partial c} \quad S_{k}^{*}=k \frac{\partial Y}{\partial k}
$$


Figure 5 shows the evolution of these parameters over time. The two curves are not correlated, which makes it possible to simultaneously identify these two parameters. However, the measurement probe is mainly sensitive to a variation of thermal conductivity. We can expect a lack of precision as to the identification of the volumetric heat capacity.

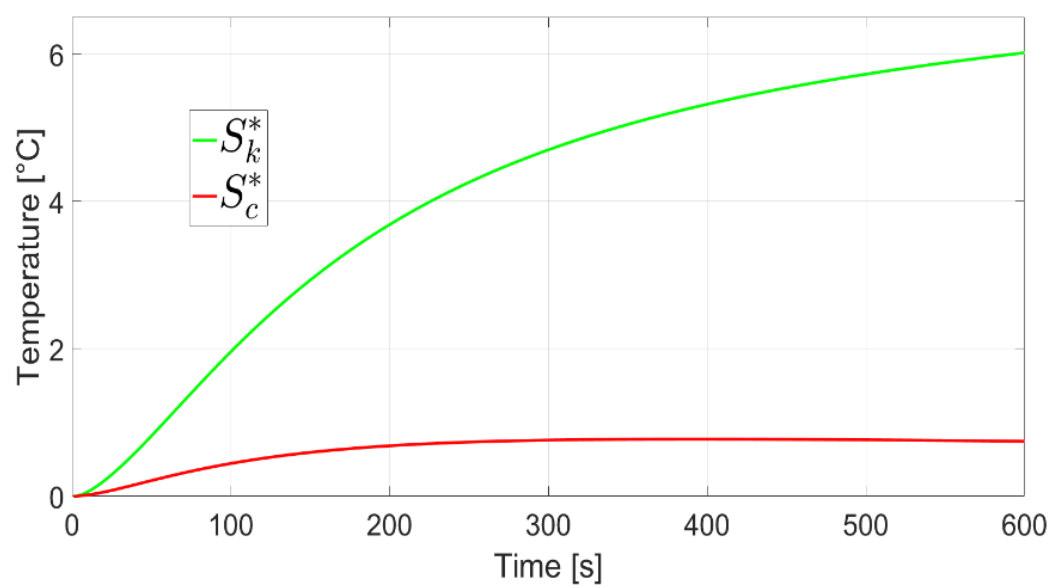

Fig. 5: Sensitivity study.

\subsection{Identification Results}

The estimation without measurement noise is performed for different reduction orders. Let us recall that the model built from $\mathrm{k}_{\text {base }}$ and $\mathrm{c}_{\mathrm{base}}$ is different from the desired pair. Table 2 summarizes the results obtained for different reduction orders as well as for the complete model. Here we note $\sigma_{k}$ and $\sigma_{c}$, as the relative errors of the average value identified, and $\sigma_{\mathrm{T}}$ as the quadratic error between the measurement and the temperature built with the parameters identified:

$$
\begin{gathered}
\sigma_{k}=\frac{\left|\hat{k}_{I}-k_{\text {exact }}\right|}{k_{\text {exact }}} \quad \sigma_{c}=\frac{\left|\hat{c}_{I}-c_{\text {exact }}\right|}{c_{\text {exact }}} \\
\sigma_{T}=\sqrt{\frac{1}{2 n_{\text {mes }}} \sum_{i=1}^{n_{\text {mes }}}\left(Y\left(t_{i}\right)-\hat{Y}\left(t_{i}, \hat{k}_{I}, \hat{c}_{I}\right)\right)^{2}}
\end{gathered}
$$

Table 2: Identification without measurement noise.

\begin{tabular}{|c|c|c|c|c|c|c|}
\hline$n$ & $\hat{k}_{I}$ & $\sigma_{k}$ & $\hat{c}_{I}$ & $\sigma_{c}$ & $\sigma_{T}$ & $\mathrm{t}_{\mathrm{cpu}}(\mathrm{s})$ \\
\hline 30 & 0,0299 & $2,3 \%$ & 40151 & $30 \%$ & 0,012 & 0,4 \\
\hline 100 & 0,0303 & $1 \%$ & 36966 & $20 \%$ & 0,008 & 2,3 \\
\hline 200 & 0,0309 & $1 \%$ & 27299 & $11,5 \%$ & 0,005 & 4,4 \\
\hline $\begin{array}{c}\text { Complete } \\
\text { model }\end{array}$ & 0,0309 & $1 \%$ & 28203 & $8.6 \%$ & 0,003 & 1105 \\
\hline
\end{tabular}

As expected, the identification of the conductivity is much more accurate than that of the volume capacity. The calculation of the temperature error recalculated with the identified parameters $\left(\sigma_{\mathrm{T}}\right)$ is very low and shows the lack of sensitivity for the temperature with respect to the volumetric capacity. The need to use a reduced model characterized by a sufficiently large order $(n=200)$ is needed to approach the value of the capacity with the same level of error as with the complete model. However, even for this reduction order, the computation time remains low compared to the time required for the complete model for the identification procedure (computing time gain equal to 250). 
Tests are carried out with two measurement noise values. $n_{I}=300$ identification tests are conducted for each of them to obtain reliable average values of the identified parameters. The results are shown in Table 3, and as histograms in Figures 6 and 7. In this table we note:

$-\bar{k}_{I}$ and $\bar{c}_{I}$ the average values of the identified parameters defined by:

$$
\bar{k}_{I}=\frac{1}{n_{I}} \sum_{i=1}^{n_{I}} \hat{k}_{I}^{i} \quad \bar{c}_{I}=\frac{1}{n_{I}} \sum_{i=1}^{n_{I}} \hat{c}_{I}^{i}
$$

- $s t d_{k}$ and $s t d_{c}$ the standard deviations that characterize the spreading of the conductivity and capacitance values that were identified during the $n_{I}$ tests (for a measurement noise $\sigma_{B}$ and reduction order $n$ fixed):

$$
s t d_{k}=\sqrt{\frac{1}{n_{I}} \sum_{i=1}^{n_{I}}\left(\hat{k}_{I}^{i}-\bar{k}_{I}\right)^{2}} \quad s t d_{c}=\sqrt{\frac{1}{n_{I}} \sum_{i=1}^{n_{I}}\left(\hat{c}_{I}^{i}-\bar{c}_{I}\right)^{2}}
$$
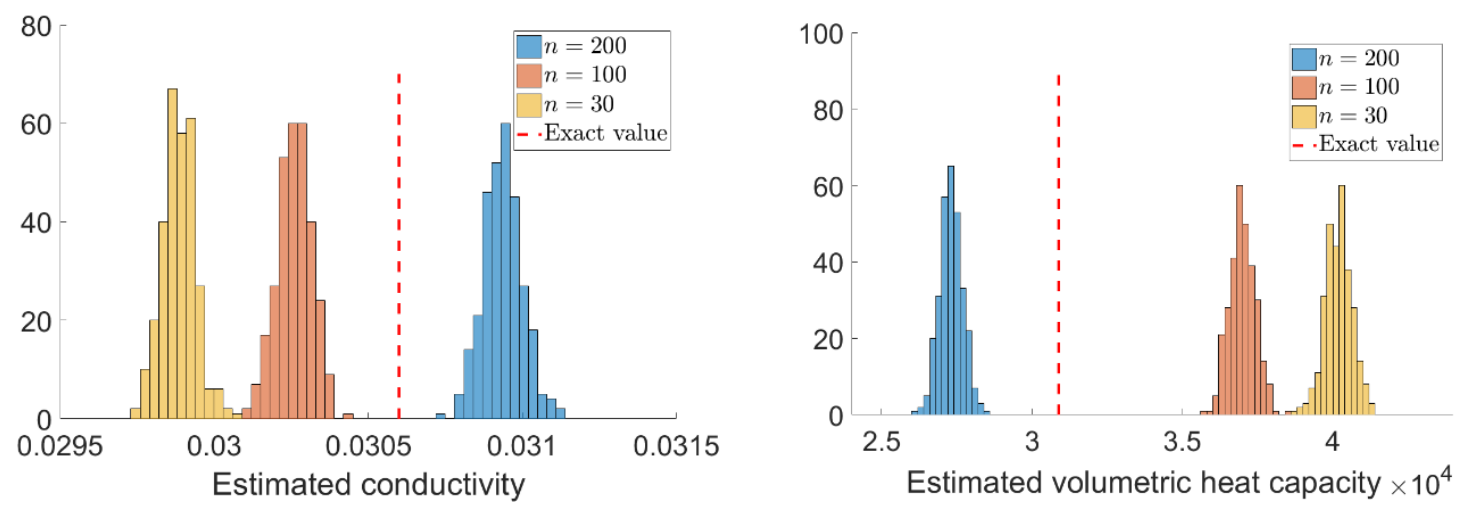

Fig. 6: Identification for a $\sigma_{\mathrm{B}}=0.05^{\circ} \mathrm{C}$ noise.
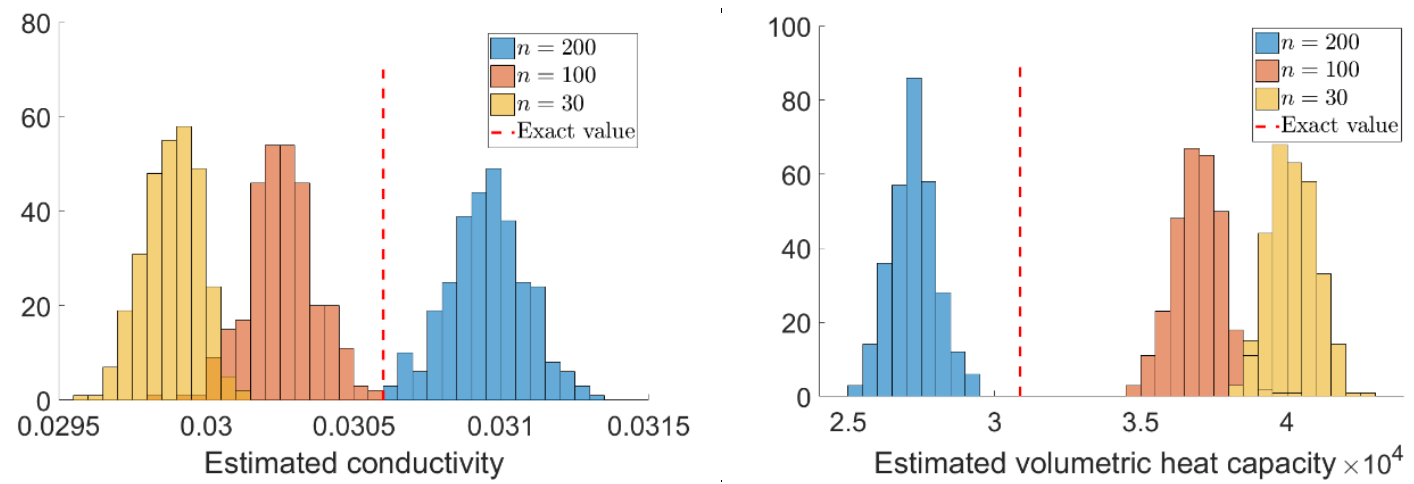

Fig. 7: Identification for a $\sigma_{\mathrm{B}}=0.1^{\circ} \mathrm{C}$ noise.

The greater the measurement noise, the more the values identified are spread over a wide range of values obtained. However, there are identical average values to the values identified without noise measurements: provided a sufficiently large number of measurements, it is possible to overcome the measurement noise. Thus, the most important parameter remains the reduction order which, given the probe's lack of sensitivity to the capacitance, causes a non-negligible error on the latter. 
It is noteworthy that the different quadratic errors of the reconstructed temperatures $\sigma_{\mathrm{T}}$ systematically correspond to the measurement noise, whatever the identification error of the volume capacity. These observations are entirely consistent with the lack of probe sensitivity with respect to the volumetric capacity, as shown in Fig. 7.

Table 3: Identification with measurement noise.

\begin{tabular}{|c|c|c|c|c|c|c|c|c|}
\hline$\sigma_{B}\left({ }^{\circ} \mathrm{C}\right)$ & $n$ & $\bar{k}_{I}$ & $s t d_{k}$ & $\sigma_{\mathrm{k}}$ & $\bar{c}_{I}$ & $s t d_{c}$ & $\sigma_{c}$ & $\sigma_{T}$ \\
\hline & 30 & 0.0299 & $0.053 .10^{-3}$ & $2.3 \%$ & 40179 & 446 & $30.1 \%$ & 0.0515 \\
\hline 0.05 & 100 & 0.0303 & $0.056 .10^{-3}$ & $1 \%$ & 36987 & 430 & $19.8 \%$ & 0.0508 \\
\hline & 200 & 0.0309 & $0.064 .10^{-3}$ & $1 \%$ & 27303 & 385 & $11.5 \%$ & 0.0502 \\
\hline & 30 & 0.0299 & $0.096 .10^{-3}$ & $2.3 \%$ & 40188 & 798 & $30.1 \%$ & 0.1007 \\
\hline 0.1 & 100 & 0.0303 & $0.110 .10^{-3}$ & $1 \%$ & 36999 & 866 & $19.8 \%$ & 0.1004 \\
\hline & 200 & 0.0310 & $0.13 .10^{-3}$ & $1.3 \%$ & 27240 & 791 & $11.8 \%$ & 0.1000 \\
\hline
\end{tabular}

\section{Conclusion}

This study has shown that an inverse procedure based on the use of a reduced numerical model can allow very fast insitu identification of thermal conductivity for insulating materials. As far as the heat capacity is concerned, given the low probe sensitivity to this parameter, a coarser estimate is possible, provided that an accurate modal reduced model is used.

These first results are promising and open to several perspectives. First of all, it must be possible to improve the procedure by integrating the reduction phase of the base in the reverse procedure. In this way, as iterations proceed, the reduced base should be better adapted to the identified parameters. Moreover, the improvement of the sensitivity of the probe to the heat capacity will require the design of a new type of probe in which the heat source and the temperature measure (s) will be separated by the insulation to characterize (bi-probes or tri-probes type model). This new type of probe can only be modelled by a 3D geometry, characterized by a large number of degrees of freedom. For such a problem, the use of a modal model will be all the more interesting.

\section{References}

[1] A. Degiovanni, "Conductivité et diffusivité thermique des solides," Techniques de 1'Ingénieur, traité Mesures et Contrôle R 2850, 1994.

[2] L. Marmoret and H. Humaish, "Limit of validity of the log-linear model for determining thermal properties of light insulation materials with cylindrical hot probe," International Journal of Thermal Sciences, vol. 117, pp. 251-259, 2017.

[3] S. Carmona, Y. Rouizi, O. Quéméner, F. Joly, "Identification de flux de chaleur, par modèle réduit combiné à la méthode de l'adjoint. Application au cas d'un disque de frein en fonctionnement," Congrès Français de Thermique, Toulouse, 2016.

[4] O. Quéméner, A. Neveu, and E. Videcoq, "A specific reduction method for the branch modal formulation: Application to a highly non-linear configuration,” International Journal of Thermal Sciences, vol. 46, pp. 890-907, 2007. 